\title{
How to prevent sports injury in amateur sports training
}

\author{
CHUAN hua Shi \\ NanTong Institute of Technology,Nantong, China, 226002 \\ qq7909558@163.com
}

Keywords: Amateur sports; common injury; cause; prevention strategy

\begin{abstract}
With the improvement of living standards, people's health awareness campaign has gradually increased, more and more people choose to carry out physical training in spare time, in order to improve body function, improve immunity, but the movement is a process of scientific system, many people are lack of scientific guidance in training, often resulted in sports injury. Light weight, skin abrasions, skeletal muscle injury, and these sports injury not only terminated the exercise, will pose a threat to the health, therefore, this article from the current status of amateur sports training of our country, combining with the common sports injury, the reasons are analyzed, and to explore the corresponding countermeasures, in order to have help to improve the overall level of amateur sports training in china.
\end{abstract}

\section{Introduction}

Sports injury is a general term for all kinds of injuries occurring in the course of sports. In recent years, with the improvement of people's material life level, as well as the national advocacy and support for lifelong sports, more and more people began to participate in amateur sports training. Sports training programs usually have a greater burden on people's muscles, bones, heart and lungs, so if you do not follow the laws of the body and the law of motion is likely to result in injury, or even damage the body function. Sports injury is different from the ordinary general industrial or daily life destroy, have a close relationship with the sports events, the training arrangement, sports environment, sports conditions and their technical movements. In the event of the sports injury, especially to have a serious impact on the physical damage, not only will the normal work, study and life of the movement, but also have some psychological pressure on it, and after the training of adverse psychological suggestion, thus affecting the enthusiasm of participating in sports training. Take into account this, in amateur sports training, the sports injury of the in-depth analysis and prevention, not only to ensure the physical health of the need, but also to eliminate their psychological barriers, improve the enthusiasm of the training needs. [1]

\section{The current status of amateur sports in China}

In our country, the concept of amateur sports training was formed in the last century in 50 s, the concept was put forward, the state has issued a "amateur sports school regulations (Draft)", "youth amateur sports school regulations (Draft)", from the amateur sports training in China gradually embarked on a scientific and standardized track. After half a century of development, amateur sports training in China has made considerable progress. The overall physical quality of the country has also been significantly improved. Although compared to the amateur sports training professional sports training, the training mechanism of low threshold, loose, but in order to ensure the diversity and effectiveness of the training, the state and society have also done a lot of investment of sports facilities, equipment, and laid a solid foundation for the cultivation of reserve talents of China's professional sports. [2]

Although China's amateur sports achievements significantly, but some problems still worth noting: first, the amateur sports training goal is relatively simple, for example in some school physical education curriculum teaching, teachers of students in the training process, more is to develop the sports skills, and ignore the sports the significance of education, thus affecting the students' 
understanding of sports training from the knowledge, skills, emotion of the three aspects of value; secondly, the lack of amateur sports training professional talents, scientific and healthy physical training needs scientific staff guidance, especially for young people in terms of physical training had just started, and the body quality is good, ready to exercise and now, if not in training teachers' professional guidance, will appear in the blind sports injury, in addition, some social needle Lack of amateur sports training organization, many sports enthusiasts, usually only yourself groping forward, and continue to accumulate experience, and it is difficult to get professional help; finally, the neglect of sports injury in sports, inevitably there will be a sports injury, but some training in sports or lack of science guide, or take unnecessary treatment measures of sports injury, affect the normal functions of the body, and this movement is obviously the result The loss outweighs the gain.

\section{Amateur sports training in the common sports injury}

Common sports injury in amateur sports training in the main are: first, muscle ligament injury, which is in motion or a sharp contraction caused by excessive tension of muscle ligament injury, the injured area there will be pain, hand can touch strip lumps obviously, serious will appear local swelling or subcutaneous hemorrhage. For example, in the movement of warm muscle ligament is not in place, lack of flexibility, if there is a greater range of motion, muscle fiber can stretch over like rubber bands as damage and even rupture; two, the ankle joint sprain of ankle joint is the most close to the ground of the weight-bearing joints, it almost takes the full weight of the body, therefore, in sport is most likely because of overload and sprain, as in basketball backboard landing angle or careless dribble and pass in football sports injury caused by improper, The main features of the lateral ankle swelling, blood stasis, pain and even varus, torn ligament or fracture dislocation, etc.; three, skin abrasions, namely skin force causes the skin tissue damage, bleeding or tissue fluid exudation, abrasions generally small, can through the body's self-healing function to heal itself, but if there is a large area of bruises, or serious damage on the skin, if you do not take debridement, disinfection and other measures, it is prone to wound infection, and tetanus; four, lumbar sprain, muscle, waist muscle, ligament and soft tissue suddenly subjected to excessive traction caused by acute laceration the external force, such as in basketball, players will jump through, dodge to pass and dribble, which is prone to lumbar sprain, lumbar The Ministry will appear after sprain local bleeding, swelling and other symptoms, even when coughing or sneezing are unbearable pain, if not to take effective measures of prevention and treatment, it is prone to lumbar muscle strain, and even affect the lumbar and lumbosacral joint; five, finger joint damage, such as in volleyball, digging, ball will cause some pressure on the finger joints, and even joint injury; six, fracture, this is a serious injury, will make the athletes in the short term can not be normal, and even affect the work and life, often said: "the one hundred day recovery period, injured in the sinews or bones" fracture is slow, if not fixed, adjustment and scientific treatment will have a serious impact on the later movement. [3]

\section{Amateur sports training in the cause of sports injury}

Lack of awareness of sports injury, lack of awareness of prevention. In amateur sports training, lack of knowledge of sports injuries, lack of self-protection awareness and prevention of injury is a major cause of bodily injury, such as some of the athletes in the movement of their physical fitness training of blind self-confidence, overload, causing muscle injury; or lack of awareness of the dangers in motion. No need to do security, causing damage to the body under the impact of the external forces; in addition, there are some amateur athletes lack of systematic learning skills of patience, in motion due to irregular movements caused by sports injuries. [4]

The preparation activities are not scientific enough. Sports may cause load and pressure, the larger of body function therefore, if not warm-up before exercise a systematic and scientific, muscle and bone is difficult to afford the desired movement strength and sensitivity, such as in the long run, if not necessary warm up, will not only weaken the endurance of athletes, but also let the cramps, sprain and other issues. At present, many athletes in amateur sports training in the warm up to take a 
perfunctory attitude, usually do a simple stretch to start the exercise, which is not in place to prepare the situation greatly improved the probability of the occurrence of sports injury.

The unreasonable arrangement of sports load. Human body exercise in unit time is limited, although through training, exercise athletes can bear will continue to increase, but if more than the load in a single movement, not only cause physical fatigue, but also the psychological state of athletes. At present, many amateur sports in our country, the athletes on their physical condition no understanding, often with a momentum overload movement, and the lack of sustained exercise stamina. In addition, in the school, some physical education teachers lack of understanding of the physical condition of the students, for a long time to turn it in the overload exercise, the body of the students also caused serious harm.

Sports venues, equipment and other objective conditions are poor. Ground and equipment is the basic condition in sports training, the support of amateur sports training is insufficient, resulting in a lack of space, equipment damage and other serious problems, which affect the smooth development of the athletes training, for example in the training, because the pitch is uneven or too hard, too slippery runway and other reasons, resulting in sports injury, or an ankle injury; in motion due to equipment obsolete, damaged, causing athletes fall, bump and other injuries, which seriously affected the enthusiasm and initiative of sports training athletes. [5]

Improper arrangement of training and competition. Sports training and competition needs to follow a certain regularity, must grasp the rhythm training, but at present in our country the amateur sports training, athletes in training and competition organization arrangements have certain shortcomings, such as schools arrange for students to participate in track and field competitions before, will make the corresponding training on it, but in in the training, teachers are lack of system, nor for the age of the students, develop training programs have gender differences, resulting in the students did not adjust to the best state in the pro game body, which not only affects the match results, but also increase the risk of injuries.

\section{Amateur sports training in how to prevent sports injury}

To strengthen the understanding and understanding of sports injury. Understanding is the action guide in amateur sports training, only to strengthen the understanding of sports injuries, to be in a position to establish a sense of security, and prepared before exercise, improve the normative action in sports, the damage appears after timely treatment. To improve the athlete's thinking, on the one hand the relevant departments should strengthen the propaganda, the common sports injuries and preventive measures, on the other hand, athletes should take the initiative to learn the knowledge of sport and health, also have an accurate judgment of their physical quality, and prevention of sports injuries for this project according to the individual.

Scientific and rational choice of training methods. The training method is to ensure a reasonable exercise effect, effective means to reduce sports injuries, in amateur sports training, adhere to the principle of first to It differ from man to man. guidance, to develop targeted programs for training the age, gender, body quality, advantages and defects, to ensure that everyone will be able to carry out professional training in sports under the guidance of science, so as to ensure the effectiveness of training; secondly, to adhere to the easy first principle, namely in the arrangement of the training load, should start from the basis of simple action to start, then gradually increase the difficulty of movement and exercise, so as to gradually improve the physical function.

Improve the warm-up and preparation before training. Warming up is an important link of sports training, to improve the warm-up and preparation before the training, athletes should first recognize the significance of warm-up and preparation activities, such as through the preparation, the body's muscle temperature will be significantly increased, the nervous system will continue to enhance the ability to work, reduce the viscosity of muscle, enzyme activity increased blood flow increased, oxygen diffusion speed, so that the body can enter into a suitable motion state; secondly, establish and perfect the warm-up, such as jogging to warm up, and then use the chest movement, abdominal movement, body movement, arm rotation movement, lateral leg stretching, lunges and 
other activities, to the body joints and ligaments muscle activity, practice, and then according to the sports for some more targeted preparations. The perfect warm-up exercise will not only make our body to exercise state, but also improve the psychological state, increase the enthusiasm of participating in sports. [6]

Improve the objective conditions of amateur sports training. The front has made certain elaboration on the relationship between sports venues, equipment and sports injuries, and to break through the venue, equipment of Amateur Sports Limited, a state, society must increase the sports infrastructure investment, such as not only to build the school playground, the purchase of equipment should be, should also increase the maintenance of investment rugged, too slippery, too much debris to clean up the site, the equipment does not meet the requirements of the timely replacement and maintenance, to ensure the use function; on the other hand, the athletes before training instructors should also carry out detailed inspection of the facilities and equipment, to ensure compliance with the movement of the corresponding standards, reduce the possibility of sports injury.

To deal with the damage occurred in a timely manner. It is important for the prevention of sports injury, but when the damage appears, how to expand the scope of treatment is also an important measure to avoid the damage in amateur sports, athletes or instructors should grasp the basic method of health care, such as in the face of the body abrasion, should be timely with saline wash the wound, wound around with $75 \%$ alcohol disinfection and if the wound is deep, serious pollution should be injected tetanus, the only way to ensure the effectiveness of prevention system. [7]

\section{Concluding remarks}

In short, in the amateur sports training is to enhance physical fitness, promote physical and mental health, but in the intense training will inevitably cause a certain impact on the body and injury, therefore, in amateur sports training, we must pay attention to the prevention of doing sports injuries, and only with strong awareness, develop a good system of preventive measures in order to have a good attitude to carry out sports, fitness and entertainment to reach.

\section{References}

[1]Zadik Y, Levin L. Does a free-of-charge distribution of boil-and-bite mouthguards to young adult amateur sportsmen affect oral and facial trauma?[J]. Dental Traumatology, 2009, 25(1):69-72.

[2]Aerts I, Cumps E, Verhagen E, et al. Efficacy of a 3 month training program on the jump-landing technique in jump-landing sports. Design of a cluster randomized controlled trial[J]. Bmc Musculoskeletal Disorders, 2009, 11(1):281.

[3]Antoni Aguiló, Pedro Tauler, Antoni Sureda, et al. Antioxidant diet supplementation enhances aerobic performance in amateur sportsmen[J]. Journal of Sports Sciences, 2007, 25(11):1203.

[4]Morel J, Combe B, Francisco J, et al. Bone mineral density of 704 amateur sportsmen involved in different physical activities.[J]. Osteoporosis International, 2001, 12(2):152-157.

[5]Dutour Geerling S, Breukelen J W M V. Analyzing aspects of coaching in amateur sport teams, and their association with team cohesion and team performance[J]. Haemophilia, 2015, 19(2):330 337.

[6]Soomro N, Sanders R, Hackett D, et al. The Efficacy of Injury Prevention Programs in Adolescent Team Sports: A Meta-analysis[J]. American Journal of Sports Medicine, 2015, 44(9):2415.

[7]Runer A, Runer F, Neunhäuserer D, et al. A 1-year prospective analysis of injuries in amateur and elite fistball.[J]. Scandinavian Journal of Medicine \& Science in Sports, 2014, 24(3):188-94. 